Check for updates

Cite this: RSC Adv., 2018, 8, 26371

\title{
A novel polysaccharide from Rhizoma panacis japonica exerts anti-inflammatory effects via STAT3 signal pathway
}

\author{
Qiu Li, (D) ${ }^{\text {b }}$ Qun Li, ${ }^{a}$ Zhihui Hao, ${ }^{d}$ Xucai Zheng ${ }^{\star c}$ and Wei He*a
}

We report a novel water-soluble polysaccharide from Rhizoma panacis japonica, namely, PJ-1 with antiinflammatory activity. We first obtained a polysaccharide from this herb by eluting with water. After fully characterizing PJ-1 with a series of chromatographic technologies, we evaluated its biological activity by stimulating Raw 264.7 cells to express inflammatory cytokines and found that PJ-1 effectively enhanced the production of anti-inflammatory factors such as IL-10. We further identified its anti-inflammatory effect in a mouse model of sepsis induced by a lipopolysaccharide. The treatment of PJ-1 evidently suppressed the secretion of major inflammatory cytokines and increased the survival rate of mice. Particularly, we verified the STAT3 signal pathway responsible for the anti-inflammatory activity. Thus, PJ-1, which has remarkable anti-inflammatory effect and mechanism of action, may be a promising candidate as an anti-inflammatory agent.

Received 5th April 2018

Accepted 4th July 2018

DOI: $10.1039 / \mathrm{c} 8 \mathrm{ra02923g}$

rsc.li/rsc-advances

Rhizoma panacis japonica, a common herbal medicine, has

\section{Introduction}

Polysaccharides derived from natural herbs are commonly considered immunomodulatory, and they exert immunoregulatory effects in vitro and in vivo. ${ }^{1-4}$ Diverse functions of natural polysaccharides such as promoting macrophage phagocytosis, ${ }^{5}$ enhancing lymphocyte functions ${ }^{6}$ and boosting anti-tumor effects $^{7}$ have been demonstrated. Among them, the interaction with receptors expressed by macrophages is a key molecular basis, which can evidently affect the functions of macrophages. ${ }^{8,9}$ For instance, dectin- $1,{ }^{10}$ mannose receptors (MR) ${ }^{11}$ and macrophage galactose-type lectin (MGL) ${ }^{12}$ specifically interplay with polysaccharides composed of mannose, glucose, and galactose, which triggers downstream signals and induces dynamic changes in cellular action. Inspired by these interactions, numerous researches have been carried out for developing polysaccharide-based macrophage-affinity therapeutic tools aimed at a series of therapeutic applications. ${ }^{9}$ As such, the screening of polysaccharides from herbs and inspection of their influences on macrophages are critical for the discovery of therapeutic agents in clinic.

\footnotetext{
${ }^{a}$ Department of Immunology, School of Basic Medical Sciences, Anhui Medical University, Hefei, China. E-mail: weihe@ahmu.edu.cn

${ }^{b}$ State Key Laboratory of Quality Research in Chinese Medicine, Institute of Chinese Medical Sciences, University of Macau, Macau SAR, China

'Department of Head, Neck and Breast Surgery, Anhui Provincial Cancer Hospital, West Branch of Anhui Provincial Hospital, The First of University of Science and Technology of China, Hefei, China.E-mail: ahszlyyzxc@163.com

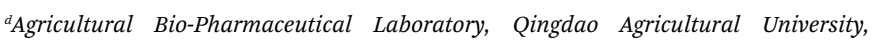
Qingdao, China. E-mail: abplab@126.com
} attracted much attention due to its various pharmacological activities recorded by traditional oriental medicine. It can 'stop bleeding', 'spread stasis', 'reduce swelling', 'relieve pain', 'stop expectorant' and 'relieve cough' in clinic. Furthermore, its versatile biological activities including anti-tumor, antiinflammatory, anti-viral, immunoregulatory and anti-fatigue activities are certified by modern pharmacology. ${ }^{13-17}$ Studies indicate that its polysaccharides may possess diverse biological activities such as anti-tumor effect $^{17}$ and anti-oxidant activity. ${ }^{18,19}$ However, it remains unclear whether the polysaccharides can interact with macrophages to modulate their action. Studies on polysaccharides against macrophages illustrate clear immunoregulatory behavior and mechanism of action.

Therefore, in this study, we isolated a water-soluble polysaccharide via ion-exchange chromatography and gel column. Then, we evaluated the macrophage-modulating activities by stimulating secretion of the inflammatory cytokine. Furthermore, we observed the relative signal pathways and verified the biological activities of the polysaccharide in vitro and in vivo. Our findings may provide a promising prospect for developing new therapeutic agents based on this active polysaccharide.

\section{Experimental}

\subsection{Materials and reagents}

Rhizoma panacis japonica was purchased from China Pharmaceutical Corporation-Canton (Guangzhou, China), and thirdparty verification was performed at Anhui Medical University. DEAE-52Cellulose and Sephadex G-100 were purchased from GE 
Healthcare (Fairfield, Connecticut, USA). Standard monosaccharides including D-glucose, L-arabinose, D-mannose $\mathrm{D}^{-}$ galactose, L-rhamnose, D-glucuronic acid and D-galacturonic acid were purchased from Sigma-Aldrich (USA). Phenol, sulfuric acid, TFA and PMP were purchased from Shanghai Aladdin Biochemical Polytron Technologies Inc. (Shanghai, China). Acetonitrile for HPLC was purchased from Merck KGaA (Darmstadt, Germany). All other chemicals and reagents used in this study were of analytical grade from Xi'an Chemical Co. (Xi'an, China). The water used was distilled water.

Murine Raw 264.7 cells, purchased from the American Type Culture Collection (ATCC), were cultured in Dulbecco's modified Eagle's medium (DMEM) with 10\% fetal bovine serum (FBS), 100 $\mathrm{U} \mathrm{mL}{ }^{-1}$ penicillin and $100 \mathrm{mg} \mathrm{mL}^{-1}$ streptomycin in $5 \% \mathrm{CO}_{2}$ at $37^{\circ} \mathrm{C}$. All cell-culture reagents were purchased from Gibco (USA). ELISA kits used for measurements of murine TNF- $\alpha$, IL- $1 \beta$ and IL10 were purchased from $4 \mathrm{~A}$ Biotech Co., Ltd (Beijing, China).

\subsection{Preparation of $\mathrm{PJ}-1$ polysaccharide}

We obtained PJ-1 polysaccharide according to our in-house protocol. ${ }^{20}$ Air-dried Rhizoma panacis japonica (500 g) was first extracted thrice with $95 \%$ ethanol for $2 \mathrm{~h}$ every time to remove fatsoluble components and organic molecules. Then, the dried residue was boiled with distilled water thrice for $3 \mathrm{~h}$ every time. The supernatant was collected, and a four-fold volume of ethanol was added and precipitated overnight at $4{ }^{\circ} \mathrm{C}$. The precipitate was collected by centrifugation (4000 rpm, $20 \mathrm{~min}$ ) at room temperature and then re-dissolved in distilled water. Proteins were removed by the Savage method (chloroform/n-butanol, $4: 1, \mathrm{v} / \mathrm{v}$ ). The collected solution was subsequently dialyzed in a dialysis bag (MW 8000-14000 Da) and lyophilized to obtain crude polysaccharide. The crude polysaccharide was then applied to a DEAE-52 column $(5 \mathrm{~cm} \times 30 \mathrm{~cm})$ eluted with distilled water $(1000 \mathrm{~mL})$ at a flow rate of $25 \mathrm{~mL} \mathrm{~h}^{-1}$. The fraction was collected and detected by the phenol-sulfuric acid method. ${ }^{21}$ This water fraction was further purified using a sephadex G-100 column $(2 \mathrm{~cm} \times 100 \mathrm{~cm})$ eluted with distilled water at a flow rate of $12 \mathrm{~mL}$ $\mathrm{h}^{-1}$ to obtain water-soluble polysaccharide $\mathrm{PJ}-1$.

\subsection{Measurement of molecular weight}

Each polysaccharide $(2.0 \mathrm{mg})$ of $\mathrm{PJ}-1$ was dissolved in water for homogeneity and molecular weight analysis using HPLC technology. ${ }^{22}$ The chromatographic conditions were as follows: Shimadzu LC20 with an RID-20 detector (Shimadzu, Japan) and

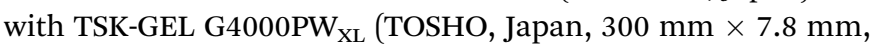
i.d.) at $35{ }^{\circ} \mathrm{C} ; 0.1 \mathrm{~N} \mathrm{NaNO}_{3}$ and $0.06 \% \mathrm{NaN}_{3}$ aqueous solutions were used as the mobile phase, and the flow rate was 0.6 $\mathrm{mL} \min ^{-1}$. Polyethyleneglycol standards with different molecular weights were used for the calibration curve.

\subsection{IR spectrum assay}

FT-IR spectrum was obtained using a PerkinElmer Spectrum 100 FT-IR Spectrometer with $\mathrm{KBr}$ pellets (PerkinElmer, Waltham, USA). PJ-1 (3 mg) was mixed with $\mathrm{KBr}$ powder, and the IR spectrum was recorded in the frequency range of 4000$450 \mathrm{~cm}^{-1}$.

\subsection{Monosaccharide composition of PJ-1}

The monosaccharide composition of PJ-1 was analyzed using the PMP pre-column derivatization method. ${ }^{20}$ Briefly, PJ-1 (4 $\mathrm{mg}$ ) was completely hydrolyzed using $2 \mathrm{~mL} 2$ M TFA in a sealed tube for $4 \mathrm{~h}$ at $110{ }^{\circ} \mathrm{C}$. Then, the products were evaporated and washed with methanol twice and dried with nitrogen to remove TFA. Hydrolysate was dissolved in water and mixed with the same volume of sodium hydroxide. Then, $100 \mu \mathrm{L}$ of $0.5 \mathrm{M}$ methanolic solution of PMP was added to the mixed solution and incubated for $120 \mathrm{~min}$ at $70^{\circ} \mathrm{C}$. Afterwards, the solution was neutralized with $0.5 \mathrm{M}$ hydrochloric acid, and chloroform (1 $\mathrm{mL}$ ) was added; the solution was vortexed fully to generate layers. The organic layer was then removed. Finally, the solution was passed through a $0.45 \mu \mathrm{m}$ membrane and analyzed by HPLC with a UV detector. It was eluted with a mixture of ammonium acetate buffer ( $\mathrm{pH} 5.5)$ and acetonitrile (82:18 v/v for $0-25 \mathrm{~min}$ and $78: 22 \mathrm{v} / \mathrm{v}$ for $25-50 \mathrm{~min})$, and the results were obtained at $245 \mathrm{~nm}$. A standard solution containing seven types of monosaccharides (mannose, rhamnose, glucuronic acid, galacturonic acid, glucose, galactose and arabinose) underwent the same protocol as described above. ${ }^{23}$

\subsection{Cell viability}

RAW 264.7 cells were cultured in 96-well plates $\left(10^{4}\right.$ per well) in DMEM medium with $10 \%$ (v/v) FBS at $37{ }^{\circ} \mathrm{C}$. After incubation for $24 \mathrm{~h}$, the cells were treated with PJ-1 polysaccharide (50-200 $\mu \mathrm{g}$ $\mathrm{mL}^{-1}$ ) for 24,48 and $72 \mathrm{~h}$. Cell viability was measured by CCK-8 assay at different time points.

\subsection{Quantitative real-time PCR (Q-PCR)}

Total RNA was obtained from the cells using TRIzol® Reagent (Invitrogen, Carlsbad, CA, USA) according to the manufacturer's instructions. The quantity and quality of total RNA were certified using Eppendorf Biophotometer Plus (Eppendorf AG, Hamburg, Germany). Q-PCR was carried out using LightCycler FastStar DNA Master SYBR Green I (Roche Diagnostics) following the protocol from the manufacturer. The fold change of each gene was normalized to that of $\beta$-actin. ${ }^{24}$ The primer sequences in this experiment are listed below (F: forward; R: reverse):

iNOS: 5'-CCAAGCCCTCACCTACTTCC-3' (F) 5'-CTCTGAGGG CTGACACAAGG-3'(R);

Arginase-1: 5'-CCAGAAGAATGGAAGAGTCAGTGT-3' (F) 5' GCAGATATGCAGGGAGTCACC-3' (R);

$\beta$-Actin: $5^{\prime}$-TGCTGTCCCTGTATGCCTCT- $3^{\prime}$ (F) and $5^{\prime}$-TTTG ATGTCACGCACGATTT-3' (R).

\subsection{Western blotting}

For Western blotting assay, cellular proteins were obtained by lysing cells in RIPA buffer containing $1 \mathrm{mM}$ phenylmethylsulfonyl fluoride (PMSF). Equal amounts of samples were subjected to SDS-PAGE electrophoresis and then transferred to PVDF membranes (Bio-Rad, USA). After blocking with skimmed milk or bovine serum albumin (5\%) for $1.5 \mathrm{~h}$ at room temperature with gentle shaking, the membranes were blotted 
with primary antibodies $(1: 1000)$ overnight at $4{ }^{\circ} \mathrm{C}$ and then exposed to secondary antibody for 2 hours at room temperature. The bands were visualized with SuperSignal West Pico Chemiluminescent Substrate (Thermo Scientific, USA). The corresponding antibodies including phosphor-STAT3, Total-STAT3 and GAPDH were obtained from cell signaling technology (Danvers, MA, USA).

\subsection{The effect of PJ-1 on macrophages}

Raw 264.7 cells were cultured and pre-treated with PJ-1 at different concentrations $\left(25-100 \mu \mathrm{g} \mathrm{mL}{ }^{-1}\right)$ for $1 \mathrm{~h}$; then, LPS (200 ng $\mathrm{mL}^{-1}$ ) was added to different groups and incubated for $20 \mathrm{~h}$ at $37^{\circ} \mathrm{C}$. Afterwards, the concentrations of TNF- $\alpha$, IL- $1 \beta$, and IL-10 in cell-culture supernatant were measured using the corresponding ELISA kits (4A Biotech Co., Ltd, Beijing, China). The operation was strictly performed according to the instructions, and the plates were read at $450 \mathrm{~nm}$ using a microplate reader (Thermo Scientific). Furthermore, total RNA was extracted, and the level of RNA including Arg1 and iNOS genes was measured according to the above-mentioned procedure. Afterwards, under the same conditions, the cells were treated in the same manner with $10 \mu \mathrm{M}$ Stattic (Sigma-Aldrich, St. Louis, Missouri, USA) as the inhibitor of the STAT3 signal pathway.

\subsection{The evaluation of PJ-1 in vivo}

Male C57BL/6 mice (18-24 g) were supplied by the Experimental Animal Centre of Anhui Medical University (Hefei, China) and randomly divided into three groups ( $n=10$ per group) including control group (saline), LPS group $\left(10 \mathrm{mg} \mathrm{kg}^{-1}\right)$, and PJ-1 (20-40 mg kg ${ }^{-1}$ ) plus LPS group (10 $\mathrm{mg} \mathrm{kg}^{-1}$ ). Particularly, PJ-1 polysaccharide was first executed by intraperitoneal injection and after $1 \mathrm{~h}$, LPS injection was administered. After eight hours, the mice with different treatments were sacrificed, and blood serum was collected. The levels of TNF- $\alpha$, IL-1 $\beta$ and IL-10 were quantified with commercial ELISA kits. The amounts and weights of surviving mice were counted at different time points. Meanwhile, the same operation was performed after inhibiting STAT3 activity with Stattic $\left(1 \mathrm{mg} \mathrm{kg}^{-1}\right)$, and the dose of PJ-1 was $20 \mathrm{mg} \mathrm{kg}^{-1}$ per mouse. Animal care and all experiments were performed according to the 'Guidelines for the Care and Use of Laboratory Animals' of Anhui Medical University.

\subsection{Statistics}

Data are presented as mean \pm standard error of the mean (SEM). Statistical analyses were performed using one-way ANOVA (GraphPad Prism 6), with * and ** standing for $P<0.05$ and $<0.01$, respectively.

\section{Results and discussion}

\subsection{Purification of crude polysaccharide}

Crude polysaccharide was isolated, followed by removal of proteins using the Sevag method. A water-soluble fraction was obtained with a DEAE-52 column and elution using distilled water. The fraction was further purified using a Sephadex G-100

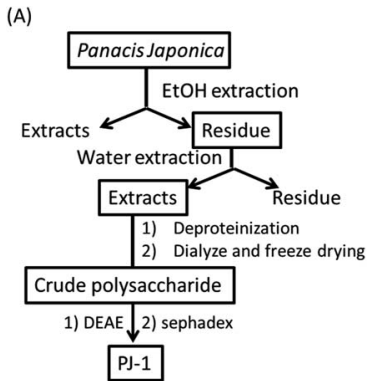

(B)

Fig. 1 (A) Scheme of PJ-1 preparation. (B) Purification of PJ-1 by DEAE column.
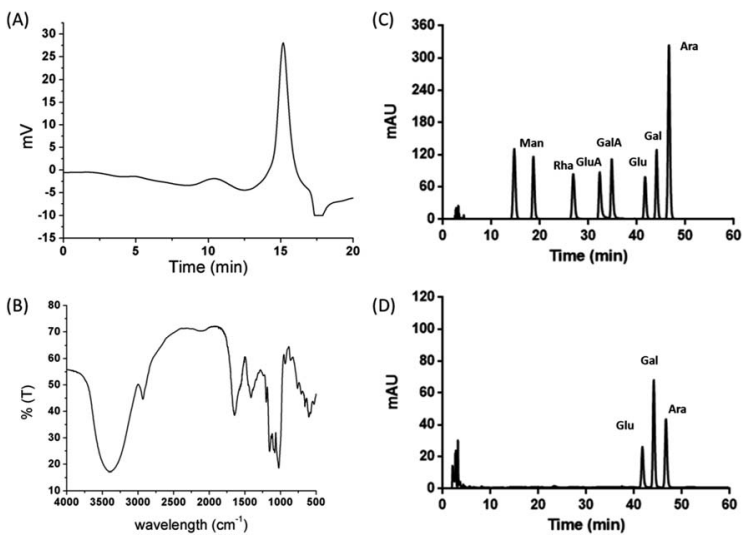

Fig. 2 Chemical analysis of PJ-1, including HPLC-GPC curve of PJ-1 (A); FT-IR spectrum (B); and PMP-derivative analyses using HPLC. (C) Standard monosaccharides and (D) monosaccharides of PJ-1.

column with distilled water as the eluent. Finally, the polysaccharide, namely, PJ-1 was obtained (Fig. 1A and B).

\subsection{Chemical analysis of $\mathbf{P J}-1$}

3.2.1 The molecular weight and FT-IR analysis of PJ-1. The homogeneity of PJ-1 was analyzed using the HPLC-RID method. After purification, PJ-1 exhibited a single peak, and its molecular weight was calculated to be about $13.6 \mathrm{kDa}$ (Fig. 2A). Meanwhile, the IR spectrum of PJ-1 showed (Fig. 2B) strong peaks at $3395 \mathrm{~cm}^{-1}, 2925 \mathrm{~cm}^{-1}$ and $1012 \mathrm{~cm}^{-1}$, which were separately assigned to the stretching vibrations of $\mathrm{O}-\mathrm{H}, \mathrm{C}-\mathrm{H}$ and $\mathrm{C}-\mathrm{O}$ bonds, respectively.

3.2.2 Monosaccharide composition of PJ-1. The monosaccharide composition of PJ-1 is critical for elucidating its biological activity. After confirming the molecular weight of $\mathrm{PJ}-1$, we further illustrated the monosaccharide content of PJ-1 using the PMP-derivative method. After the hydrolysis and derivation of PJ-1, the sample was subjected to HPLC analysis; standard monosaccharides were also subjected to the same method (Fig. 2C and D). The results suggested that PJ-1 consisted of glucose, galactose and arabinose with a molar ratio of 1 : 2.4 : 1.6.

\subsection{Biological activity of $\mathrm{PJ}-1$ in vitro}

3.3.1 PJ-1 influences secretion of cytokines in macrophage. Having verified the molecular weight and monosaccharide 
composition of PJ-1 polysaccharide, we further inspected its biological activity. First, cell viability assay was performed, and the results showed that $\mathrm{PJ}-1$ polysaccharide had no significant toxicity to macrophages (Fig. 3A). Subsequently, the effect of PJ1 on macrophages was evaluated, and the secretion of cytokines and the level of RNA were measured. The results suggested that PJ-1 significantly decreased the level of iNOS and increased the level of Arg-1 when the concentration of PJ-1 was increased (Fig. 3B and C). Meanwhile, PJ-1 could inhibit the secretion of inflammatory factors including TNF- $\alpha$ and IL-1 $\beta$ while promoting the secretion of IL-10 with the increasing dose of PJ-1 (Fig. 3D-F). In addition, we noticed that PJ-1 without the stimulation of LPS could not induce any marked change in cytokines and related gene expressions (data not shown). Together, it was confirmed that $\mathrm{PJ}-1$ exhibited efficient anti-inflammatory effect in vitro.

3.3.2 PJ-1 affects macrophage via STAT-3 signal pathway. To explore the underlying mechanism of PJ-1's antiinflammatory activity, we tested the effects of PJ-1 on the activation of signal transducers and activators of transcription 3 (STAT3) pathways. STAT3, a member of the STAT family of transcription factors, possesses key functions in development
(A)

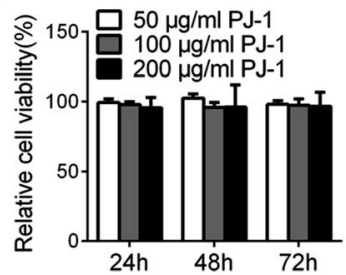

(C)

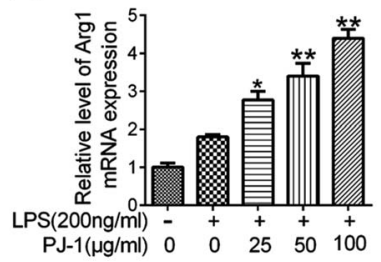

(E)

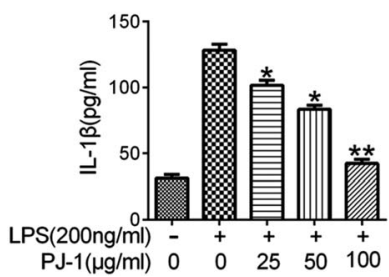

(B)

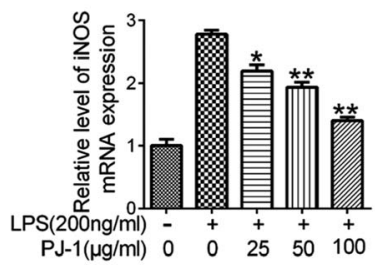

(D)

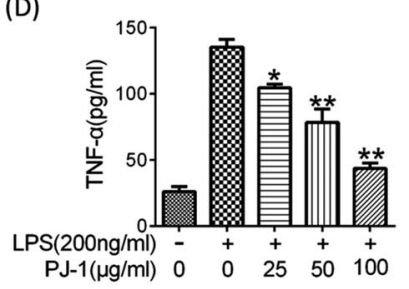

(F)

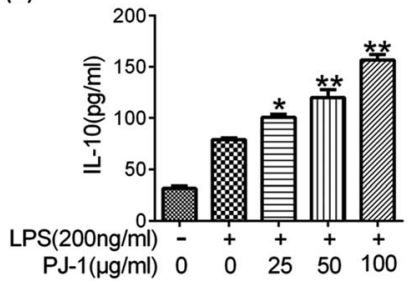

Fig. 3 Effects of PJ-1 on macrophage viability and phenotype. (A) Viability of Raw 264.7 cells treated with different doses of PJ-1 (50$200 \mu \mathrm{g} \mathrm{mL}^{-1}$ ) for 24,48 and $72 \mathrm{~h}$. The data were normalized to PBStreated cells. Raw 264.7 cells were pre-incubated with different doses of PJ-1 for $1 \mathrm{~h}$ and treated with LPS (200 $\left.\mathrm{ng} \mathrm{mL}^{-1}\right)$ for $20 \mathrm{~h}$. The mRNA levels of iNOS and Arg1 were examined by $Q-P C R(B$ and $C)$ and the protein levels of TNF- $\alpha, I L-10$ and IL-1 $\beta$ in the supernatants (D-F) were tested by ELISA. The results represent 3 independent experiments and are expressed as mean \pm SEM. $*,<0.05$ and $* *,<0.01$ compared with the LPS group. and tissue homeostasis. ${ }^{25,26}$ It has been reported that activated STAT3 can promote macrophage to M2 phenotype differentiation. ${ }^{27-29}$ Western blotting analysis was performed in PJ-1treated Raw 264.7. The results indicated that PJ-1 could promote STAT3 phosphorylation in a dose-dependent manner in Raw 264.7 (Fig. 4A). Subsequently, we used an STAT3 inhibitor (Stattic) to assess the role of STAT3 in the anti-inflammatory activity of PJ-1. The data showed no marked change in the levels of cytokines (TNF- $\alpha$, il-10 and IL-1 $\beta$ ) (Fig. 4B, D and E), and related gene mRNA expressions (iNOS and Arg1) (Fig. 4C and F) were observed in the PJ-1 + Stattic group with LPS stimulation compared with the observations for the Stattic + LPS group. Analogously, the results of Western blotting showed that under
(A)

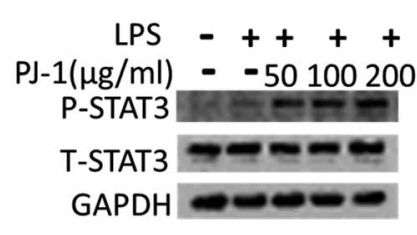

(C)

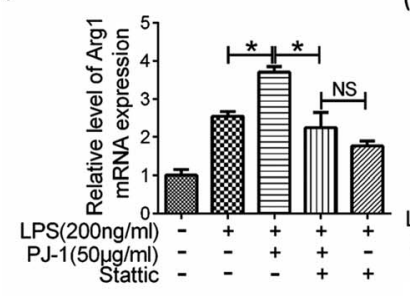

(E)

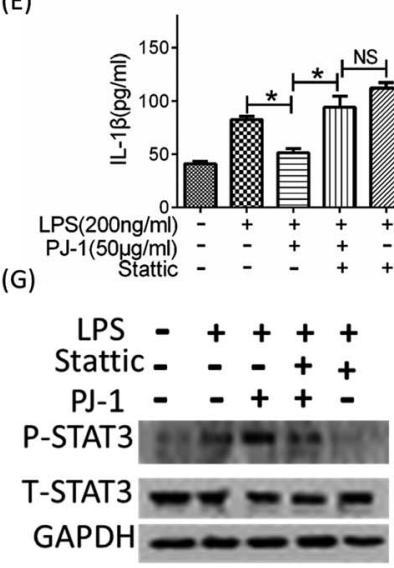

(B)

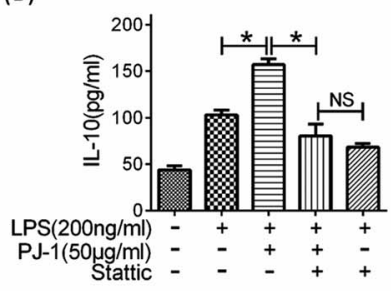

(D)

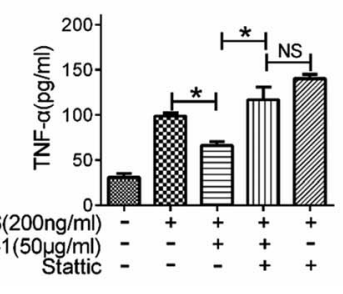

(F)

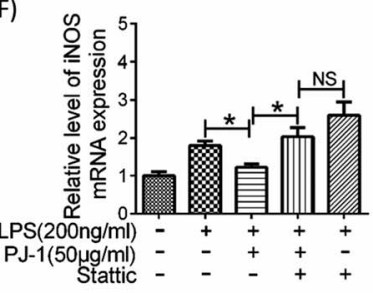

Fig. 4 PJ-1 regulated macrophage phenotype by enhancing STAT3 activation. (A) Expressions of phosphorylated-STAT3 (P-STAT3) and total-STAT3 (T-STAT3) evaluated by Western blotting. (B-F) Macrophages were pre-incubated in $10 \mu \mathrm{M}$ Stattic for $30 \mathrm{~min}$, followed by co-treatment with PJ-1 and Stattic for $1 \mathrm{~h}$ and then, they were cultured with LPS (200 $\mathrm{ng} \mathrm{mL}^{-1}$ ) for $24 \mathrm{~h}$. The supernatants were collected to determine TNF- $\alpha, \mathrm{IL}-10$ and IL-1 $\beta$, and gene iNOS and Arg1 expressions were tested by Q-PCR. (G) Macrophages were pre-incubated in $2 \mu$ M Stattic for 30 min, followed by co-treatment with PJ-1 and Stattic for $1 \mathrm{~h}$ and then, they were cultured with LPS $\left(200 \mathrm{ng} \mathrm{mL}^{-1}\right)$ for $30 \mathrm{~min}$; the expressions of P-STAT3 and T-STAT3 were evaluated by Western blotting analysis. The results represent 3 independent experiments and are expressed as mean \pm SEM. *,$<0.05$ compared with LPS group or LPS + PJ-1 group and NS compared with LPS + Stattic group. 
treatment of LPS, PJ-1 and Stattic co-treatment decreased the phosphorylation level of STAT3 relative to PJ-1 treatment alone (Fig. 4G). These results suggested that the anti-inflammatory effects of PJ-1 in macrophages depended on the activation of STAT3.

\subsection{Anti-inflammatory activity of $\mathrm{PJ}-1$ in vivo}

3.4.1 The survival rate and body weight of mice. To further evaluate the anti-inflammatory effect of PJ-1, we constructed a sepsis model in mice during which macrophages played vital roles in inducing inflammation. As such, we established the sepsis model in mice using LPS injection and tested the antiinflammatory effect of PJ-1. After the injection of LPS, all mice had septic shock within $60 \mathrm{~h}$ and the group with PJ-1 treatment showed higher survival rate than the other groups. When the mice with LPS treatment died, the PJ-1 group (40 $\mathrm{mg} \mathrm{kg}{ }^{-1}$ ) exhibited survival rate of approximately 60\% (Fig. 5A). Meanwhile, the body weight of the PJ-1 group (40 $\mathrm{mg} \mathrm{kg}^{-1}$ ) first decreased after LPS injection and then increased slowly (Fig. 5B). The results suggested that PJ-1 showed prominent anti-inflammatory effect.

3.4.2 Measurement of cytokines in serum. To further assess whether PJ-1 can alleviate inflammatory response, cytokines including TNF- $\alpha$, IL-1 $\beta$ and IL-10 of different groups in serum were measured at $8 \mathrm{~h}$. The results showed that two major inflammatory cytokines TNF- $\alpha$ and IL-1 $\beta$ evidently decreased and IL-10, an anti-inflammatory factor, markedly increased in mice pre-treated with $\mathrm{PJ}-1$ at each concentration, compared to the group treated with LPS only (Fig. 5C-E).

\subsection{The anti-inflammatory effect of PJ-1 in vivo depends on STAT3 activation}

We pre-treated LPS-challenged mice with Stattic to further investigate the role of STAT3 activation on the effect of PJ-1 on sepsis in vivo. Co-treatment with Stattic and PJ-1 could eliminate the therapeutic effect of $\mathrm{PJ}-1$ including recovery of losses in body weight and enhancement of survival rates (Fig. 6A and B).
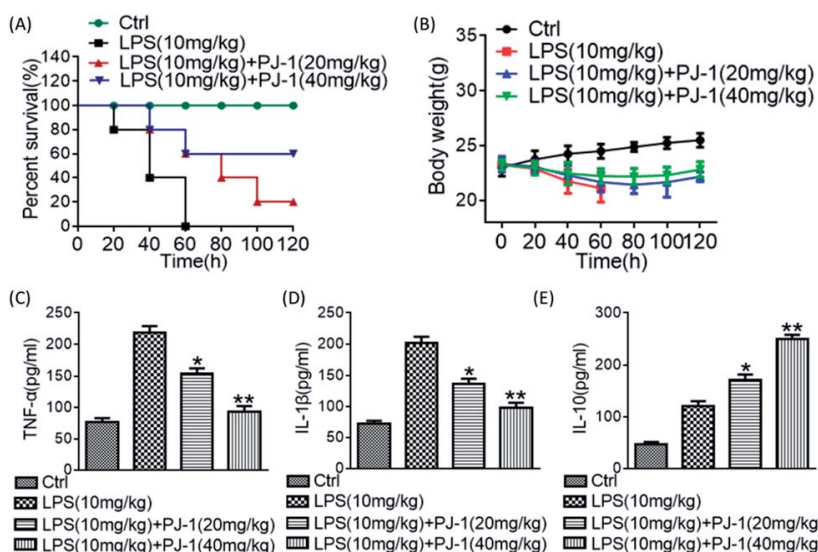

(E)

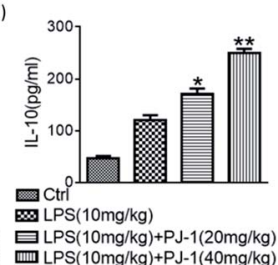

Fig. 5 PJ-1-protected mice against LPS-induced sepsis. The survival rate (A) and body mass of mice (B). The effect of PJ-1 on serum cytokine levels of TNF- $\alpha, I L-1 \beta$ and IL-10 in LPS-challenged mice (CE). Values are expressed as mean $\pm \operatorname{SEM}(n=10$ mice per group). *, $<0.05$ and $* *,<0.01$ compared with LPS group.
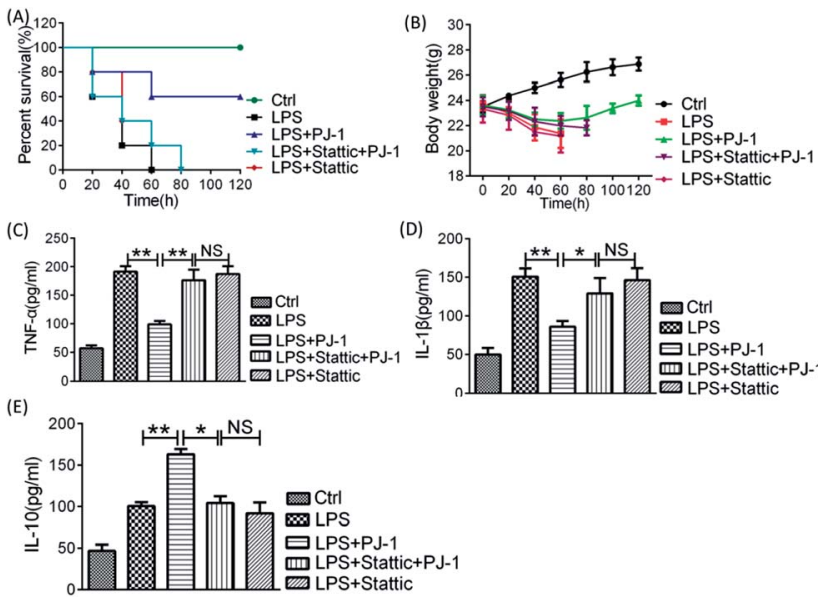

Fig. 6 The therapeutic effect of PJ-1 was eliminated by the STAT3 inhibitor Stattic. The effect of Stattic on PJ-1-treated mice with sepsis was assessed based on the survival rate $(A)$ and body weight changes (B). The effect of Stattic on the serum cytokine levels of TNF- $\alpha, I L-1 \beta$ and IL-10 in PJ-1-treated mice with sepsis (C-E). Values are expressed as mean $\pm \operatorname{SEM}(n=10$ mice per group). $* *,<0.01$ compared with LPS group; NS compared with LPS + Stattic + PJ-1 group.

Meanwhile, co-treatment with Stattic and PJ-1 eliminated the anti-inflammatory effect of PJ-1 on mice with sepsis (Fig. 6C-E). These results suggested that the anti-inflammatory effect of PJ-1 in vivo may be mediated by STAT3 activation.

\section{Conclusions}

In this study, we obtained a water-soluble polysaccharide, PJ-1, which can modulate the function of macrophages. We characterized its molecular weight and monosaccharide composition and further validated the anti-inflammatory effect of this polysaccharide in vitro and in vivo. The results obtained indicated that PJ-1 could effectively suppress the secretion of major inflammatory cytokines. Moreover, we certified its mechanism of action with multiple experiments and confirmed that it exerted anti-inflammatory effect via the STAT3 signal pathway. Taken together, PJ-1, examined by a series of evaluations, has remarkable anti-inflammatory effect in vitro and in vivo. Therefore, we assume that PJ-1 has a promising prospect of becoming an outstanding candidate as an anti-inflammatory agent.

\section{Conflicts of interest}

There are no conflicts to declare.

\section{Abbreviations}

PJ

TFA

PMP

UV

IR
Rhizoma panacis japonica

Trifluoroacetic acid

1-Phenyl-3-methyl-5-pyrazolone Ultraviolet

Infrared 


\section{Acknowledgements}

This work was supported by Scientific Research of BSKY (XJ201726) from Anhui Medical University, Anhui Institute of Translational Medicine Research Fund (2017zhyx10), and funding from the Qingdao Science and Technology Program (15-10-3-15-(41)-zch). All animal procedures were performed in accordance with the Guidelines for Care and Use of Laboratory Animals of Anhui Medical University and experiments were approved by the Animal Ethics Committee of Anhui Medical University.

\section{Notes and references}

1 Q. Li, Y. Feng, W. He, L. Wang, R. Wang, L. Dong and C. Wang, Carbohydr. Polym., 2017, 169, 304-314.

2 Y.-Q. Du, Y. Liu and J.-H. Wang, Int. J. Biol. Macromol., 2015, 72, 1272-1276.

3 Q. Fang, J.-F. Wang, X.-Q. Zha, S.-H. Cui, L. Cao and J.-P. Luo, Carbohydr. Polym., 2015, 134, 66-73.

4 F. Kallel, D. Driss, F. Bouaziz, L. Belghith, S. Zouari-Ellouzi, A. Haddar, S. E. Chaabouni and R. Ghorbel, $R S C A d v$, 2015, 5, 6728-6741.

5 X. Chu, X.-J. Liu, J.-M. Qiu, X.-L. Zeng, H.-R. Bao and J. Shu, Environ. Toxicol. Pharmacol., 2016, 48, 76-84.

6 Q.-D. Xiang, Q. Yu, H. Wang, M.-M. Zhao, S.-Y. Liu, S.-P. Nie and M.-Y. Xie, J. Agric. Food Chem., 2017, 65, 5306-5315.

7 A. Zong, Y. Liu, Y. Zhang, X. Song, Y. Shi, H. Cao, C. Liu, Y. Cheng, W. Jiang and F. Du, Carbohydr. Polym., 2015, 129, 50-54.

8 S. S. Ferreira, C. P. Passos, P. Madureira, M. Vilanova and M. A. Coimbra, Carbohydr. Polym., 2015, 132, 378-396.

9 S.-Z. Xie, R. Hao, X.-Q. Zha, L.-H. Pan, J. Liu and J.-P. Luo, Carbohydr. Polym., 2016, 146, 292-300.

10 C. Deng, H. Fu, J. Shang, J. Chen and X. Xu, Int. J. Biol. Macromol., 2018, 109, 369-374.

11 W.-J. Li, X.-F. Tang, X.-X. Shuai, C.-J. Jiang, X. Liu, L.-F. Wang, Y.-F. Yao, S.-P. Nie and M.-Y. Xie, J. Agric. Food Chem., 2017, 65, 348-357.

12 I. G. Zizzari, P. Martufi, F. Battisti, H. Rahimi, S. Caponnetto, F. Bellati, M. Nuti, A. Rughetti and C. Napoletano, PLoS One, 2015, 10, e0132617.
13 D. Xukun, M. Xue, C. Jian, Y. Wenzhong, L. You, Z. Biqun, J. Jie, C. Shuang and W. Fei, J. South-Cent. Univ. Natl., Nat. Sci. Ed., 2013, 1, 011.

14 T. Wang, Y. Dai, Y. Dun, C. Zhang, J. Wan, L. Deng, Z. Zhou, C. Liu and D. Yuan, Immunopharmacol. Immunotoxicol., 2014, 36, 404-411.

15 X. Yang, R. Wang, S. Zhang, W. Zhu, J. Tang, J. Liu, P. Chen, D. Zhang, W. Ye and Y. Zheng, Carbohydr. Polym., 2014, 101, 386-391.

16 B. Klimova and K. Kuca, Curr. Alzheimer Res., 2017, 14, 680685.

17 C. Chen, W. Wu, X. Xu, L. Zhang, Y. Liu and K. Wang, Carbohydr. Polym., 2014, 105, 308-316.

18 X. Yang, R. Wang, S. Zhang, W. Zhu, J. Tang, J. Liu, P. Chen, D. Zhang, W. Ye and Y. Zheng, Carbohydr. Polym., 2014, 101, 386-391.

19 H. Zhang, H. Zhao, X. Zhou, X. Yang, S. Shen, J. Wang, Z. Wang and L. Geng, RSC Adv., 2016, 6, 110706-110721.

20 Q. Li, G. Guo, F. Meng, H. H. Wang, Y. Niu, Q. Zhang, J. Zhang, Y. Wang, L. Dong and C. Wang, ACS Macro Lett., 2016, 5, 617-621.

21 M. Dubios, K. A. Gilles, J. K. Hamilton, P. A. Rebers and F. Smith, Anal. Chem., 1956, 28, 350-356.

22 K. L. Cheong, D. T. Wu, J. Zhao and S. P. Li, J. Chromatogr. A, 2015, 1400, 98-106.

23 F.-C. Meng, C. Yuan, X.-J. Huang, W.-J. Wang, L.-G. Lin, X.-T. Zhang, H.-Y. Jiao and Q.-W. Zhang, Phytochem. Lett., 2016, 15, 108-112.

24 W. He, Y. Zhu, R. Mu, J. Xu, X. Zhang, C. Wang, Q. Li, Z. Huang, J. Zhang and Y. Pan, Biochem. Pharmacol., 2017, 145, 132-146.

25 H. F. Zhang and R. Lai, Cancers, 2014, 6, 1408-1440.

26 D. E. Levy and C. K. Lee, J. Clin. Invest., 2002, 109, 1143-1148.

27 C. Qin, W. H. Fan, Q. Liu, K. Shang, M. Murugan, L. J. Wu, W. Wang and D. S. Tian, Stroke, 2017, 48, 3336-3346.

28 H. S. Chung, B. S. Lee and J. Y. Ma, J. Evidence-Based Complementary Altern. Med., 2017, 2017, 4218468.

29 H. Zhao, Q. Shang, Z. Pan, Y. Bai, Z. Li, H. Zhang, Q. Zhang, C. Guo, L. Zhang and Q. Wang, Diabetes, 2018, 67, 235-247. 